\title{
Dieta da pirambeba Serrasalmus brandtii Reinhardt (Teleostei, Characidae) em quatro lagoas marginais do rio São Francisco, Brasil
}

\section{Paulo dos Santos Pompeu ${ }^{1}$}

\begin{abstract}
Diet of pirambeba Serrasalmus brandtii Reinhardt (Teleostei, Characidae) in four floodplain lakes in São Francisco river, Brazil. The diet of pirambeba Serrasalmus brandtii Reinhardt 1874 was studied in four floodplain lakes in São Francisco river. The main food items consumed were chunks of fish flesh, small fishes, scales, fins and aquatic insects. Trophic ontogeny was observed in all lakes. The amount of fishes in the diet of $S$. brandtii increased with the length of the individuals, while the amount of aquatics insects, fins and scales decreased. Aquatic insects were consumed in greater proportion during the wet season. Fish ingestion, including scales and fins, was correlated with the abundance of fishes.

KEY WORDS. Serrasalmus brandtii, diet, floodplain lakes, São Francisco River
\end{abstract}

A subfamília Serrasalminae inclui piranhas e pirambebas e constitui um grupo de peixes com dieta, tática de caça e comportamento social bastante diversificados (Goulding 1980; BRAGA 1981; NICO \& TAPHORN 1988; SAZIMA \& MACHADO 1990).

Na América do Sul, os hábitos alimentares destes peixes foram estudados para espécies dos Llanos da Venezuela (MACHADO-ALlisON \& GARCIA 1986; NICO \& TAPHORN 1988; Winemiller 1989), da Guyana (PAULY 1994), do Pantanal Matogrossense (SAZIMA \& MACHADO 1990), da bacia Amazônica (Goulding 1980; GoULDING et al. 1988; LEÃo et al. 1991) e nordeste do Brasil (BRAGA 1981).

Na bacia do rio São Francisco ocorrem duas espécies de Serrasalminae: a piranha vermelha Pygocentrus piraya (Cuvier, 1820) e a pirambeba Serrasalmus brandtii Reinhardt, 1874. Embora a pirambeba represente geralmente uma grande nercentagem da biomassa de peixes em suas lagoas marginais (BRAGA 1964), não sxistem estudos sobre a alimentação desta espécie nestes ambientes.

Este trabalho teve como objetivo determinar a dieta da pirambeba (Serrasalmus brandtii) em quatro lagoas marginais do rio São Francisco, relacionando sua dieta à disponibilidade de alimento.

\section{MATERIAL E MÉTODOS}

\section{Área de estudo}

A bacia do rio São Francisco, com área de $631.133 \mathrm{~km}^{2}$, corresponde a 7,4\% do território brasileiro. Situa-se entre os paralelos $21^{\circ}$ e $7^{\circ} \mathrm{S}$, o que the confere características climáticas bastante variadas. As precipitações variam entre $350 \mathrm{~mm}$

1) Departamento de Zoologia, Instituto de Ciências Biológicas, Universidade Federal de Minas Gerais. Avenida Antônio Carlos 6627. 31270-901 Belo Horizonte, Minas Gerais, Brasil.

E-mail: pompeups@hotmail.com 
e $1900 \mathrm{~mm}$ em anos normais, e seu regime hidrológico caracteriza-se por apresentar cheias no verão (novembro a março) e estiagem no inverno (junho a outubro) (PlanVASF 1986).

As lagoas estudadas loçalizam-se na região do médio São Francisco, nos municípios de Jaíba (lagoas Juazeiro e Cajueiro), Itacarambí (lagoa Curral de Vara) e no alto São Francisco no munícipio de Lagoa da Prata (lagoa Feia), Minas Gerais. Em anos normais, grande parte das lagoas marginais do rio São Francisco são inundadas. O período de estudo (setembro de 1994 a março de 1996), no entanto, foi caracterizado pela ausência de cheias, sendo que nenhuma das quatro lagoas se comunicou com o rio São Francisco.

As lagoas Feia e Cajueiro se caracterizaram por possuírem água clara e grande quantidade de macrófitas submersas e flutuantes. Já as lagoas Curral de Vara e Juazeiro apresentaram águas mais turvas e leito sem macrófitas submersas. O tamanho das lagoas sofreu grande variação durante o período de estudo, sendo que, para todas elas, a área oscilou de 0,20 a $0,50 \mathrm{~km}^{2}$ e a profundidade de 1 a $5 \mathrm{~m}$.

Durante o período de setembro de 1994 a setembro de 1995, a temperatura da água nas lagoas variou de 24,0 a $30,1^{\circ} \mathrm{C}$, o $\mathrm{pH}$ de 6,7 a 8,3 , a condutividade de 95 a $360 \mu \mathrm{S} / \mathrm{cm}$ e o oxigênio dissolvido de 5,0 a $7,2 \mathrm{mg} / \mathrm{l}$.

\section{Métodos}

As lagoas Curral de Vara, Cajueiro e Juazeiro foram amostradas por um período de dois anos, durante os quais foram realizadas quatro coletas: duas ao final da estação seca (set/94 e set/95) e duas ao final da estação chuvosa (mar/95 e mar/96). Na lagoa Feia foram realizadas coletas no final da estação seca (ago/95) e início da estação chuvosa (nov/95).

Em todas as lagoas, os peixes foram capturados através de redes de emalhar com tamanhos de malha de 3, 4, 5, 6, 7, 8, 10, 12, 14 e $16 \mathrm{~cm}$ (medidos entre nós opostos), que foram armadas de 10:00 às 14:00 e das 18:00 às 22:00 horas. Todos os peixes capturados foram separados por malha e imediatamente fixados em formalina $10 \%$. No laboratório, todos os exemplares capturados foram pesados, medidos (comprimento padrão - CP) e posteriormente conservados em solução de álcool $70^{\circ} \mathrm{GL}$.

Todos os exemplares de Serrasalmus brandtii foram dissecados para a análise do conteúdo estomacal. Os itens alimentares foram pesados (precisão de $0,01 \mathrm{~g}$ ) e identificados até a menor categoria taxonômica possível. Foram então determinados a frequeiência de ocorrência $(\mathrm{F} i)$ e o peso relativo de cada item $(\mathrm{P} i)$. Para a verificação de ontogenia trófica os exemplares foram separados em três classes de tamanho $(\mathrm{CP}<8 \mathrm{~cm}, 8 \mathrm{~cm} \leq \mathrm{CP}<16 \mathrm{~cm}$ e $\mathrm{CP} \geq 16 \mathrm{~cm})$.

O índice alimentar, proposto por KAWAKAMI \& VAZZOLER (1980), foi então calculado para cada lagoa, coleta e classe de tamanho segundo a fórmula:

$$
I A i=\frac{(F i \cdot P i)}{\sum_{i=1}^{n} F i \cdot P i}
$$

onde: $\left(\mathrm{IA}_{i}\right)$ índice alimentar do item $i,\left(\mathrm{~F}_{i}\right)$ freqüência de ocorrência do item $i,\left(\mathrm{P}_{i}\right)$ peso do item $i$. 
Para cada lagoa calculou-se, por coleta, as capturas por unidade de esforço em número (CPUEn) segundo a fórmula:

$$
\text { CPUEn } \sum_{m=3}^{12}\left(\frac{N m}{E P m}\right) \cdot 100
$$

onde: (CPUE $)_{n}$ captura em número por unidade de esforço, $\left(\mathrm{N}_{\mathrm{m}}\right)$ número total dos peixes capturados na malha $\mathrm{m},(\mathrm{Epm})$ esforço de pesca, que representa a área em $\mathrm{m}^{2}$ das redes de malha $\mathrm{m},(\mathrm{m})$ tamanho da malha $(3,4,5,6,7,8,10$ e $12 \mathrm{~cm})$.

Para determinar a relação entre a dieta de $S$. brandtii e a abundância de alimentos, foi testada, através de regressão linear, a relação entre a freqüência de ocorrência (Fi) de peixes, escamas e nadadeiras no conteúdo estomacal e a abundância de peixes das lagoas (CPUEn). O consumo de insetos aquáticos (IA $i$ ), por outro lado, foi comparado entre as estações seca e chuvosa.

\section{RESULTADOS}

Foram coletados 357 exemplares de S. brandtii, sendo 150 na estação seca e 207 na chuvosa. Dos exemplares capturados, em 212 (59\%) foi encontrado algum conteúdo estomacal.

Os principais itens alimentares foram peixes (pedaços ou inteiros), nadadeiras, escamas e insetos aquáticos. Matéria vegetal, moluscos e zooplâncton foram consumidos em menor proporção (Tab. I). Os vegetais consumidos foram representados por macrófitas e algas filamentosas e os moluscos por gastrópodes e bivalves. Os insetos aquáticos mais consumidos foram representados, em ordem decrescente, pelas ordens Diptera, Odonata, Hemiptera, Trichoptera e Ephemeroptera. Foram identificadas um total de 11 espécies de peixes no conteúdo estomacal de S. brandtii (Tab. II), uma boa parte representada por characídeos de pequeno porte que foram ingeridos inteiros.

Tabela I. Itens alimentares consumidos por Serrasalmus brandtii por lagoa e estação. (N) Número de individuos com algum conteúdo estomacal; índice alimentar: $(-)$ menor que $10 \%$, $(+)$ entre 10 e $30 \%,\left(^{++}\right.$entre 30 e $60 \%,\left(^{+++}\right.$maior que $60 \%$.

\begin{tabular}{|c|c|c|c|c|c|c|c|c|c|}
\hline \multirow{2}{*}{ Lagoas } & \multirow{2}{*}{ Periodo } & \multirow{2}{*}{$\mathrm{N}$} & \multicolumn{7}{|c|}{ Itens alimentares } \\
\hline & & & $\begin{array}{c}\text { Insetos } \\
\text { aquáticos }\end{array}$ & Escamas & Nadadeiras & Peixes & $\begin{array}{l}\text { Matéria } \\
\text { vegetal }\end{array}$ & Moluscos & Zooplâncton \\
\hline \multirow[t]{2}{*}{ Curral de Vara } & Seca & 40 & - & + & + & + & & & \\
\hline & Chuva & 49 & + & - & - & + & - & & \\
\hline \multirow[t]{2}{*}{ Cajueiro } & Seca & 6 & - & + & & + & & & \\
\hline & Chuva & 21 & + & + & - & - & & - & \\
\hline \multirow[t]{2}{*}{ Juazeiro } & Seca & 12 & & + & + & - & - & & \\
\hline & Chuva & 19 & & - & - & +++ & - & & - \\
\hline \multirow[t]{2}{*}{ Feia } & Seca & 32 & + & + & - & + & - & & \\
\hline & Chuva & 33 & +++ & - & - & + & - & & \\
\hline
\end{tabular}

Embora o número de presas identificadas tenha representado apenas de 6 a $20 \%$ da riqueza total registrada nas lagoas (Tab. II), estas espécies corresponderam a mais de $70 \%$ das capturas em número nestes ambientes (Fig. 1).

A distribuição dos indivíduos com conteúdo estomacal por classe de tamanho foi a mesma nas duas estações (Fig. 2). 
Tabela II. Espécies de peixes identificadas no conteúdo estomacal de Serrasalmus brandtii por lagoa.

\begin{tabular}{|c|c|c|c|c|}
\hline \multirow{2}{*}{ Espécies } & \multicolumn{4}{|c|}{ Lagoas } \\
\hline & Cajueiro & Juazeiro & Curral de Vara & Feia \\
\hline \multicolumn{5}{|l|}{ Characiformes } \\
\hline \multicolumn{5}{|l|}{ Characidae: Tetragonopterinae } \\
\hline Astyanax bimaculatus (Reinhardt, 1874) & & $x$ & & \\
\hline Hemigrammus marginatus Ellis, 1911 & & & $x$ & \\
\hline Moenkhausia costae (Steindachner, 1907) & & $\mathrm{x}$ & & \\
\hline Tetragonopterus chalceus Agassiz, 1829 & $\mathrm{x}$ & & $\mathrm{x}$ & \\
\hline \multicolumn{5}{|l|}{ Characidae: Triportheinae } \\
\hline Triportheus guentheri (Garman, 1890) & & $\mathrm{x}$ & & \\
\hline \multicolumn{5}{|l|}{ Characidae: Serrasalminae } \\
\hline Serrasalmus brandtii Reinhardt, 1874 & & & & $x$ \\
\hline \multicolumn{5}{|l|}{ Characidae: Anostomidae } \\
\hline Leporinus renhardti Lütken, 1874 & $\mathrm{x}$ & & $\mathrm{x}$ & \\
\hline Schizodon knerii (Steindachner, 1875) & $x$ & $\mathrm{x}$ & & \\
\hline \multicolumn{5}{|l|}{ Characidae: Curimatidae } \\
\hline Curimatella lepidura Eig. \& Eigenmann, 1889 & & $\mathrm{x}$ & $x$ & $\mathrm{x}$ \\
\hline \multicolumn{5}{|l|}{ Siluriformes } \\
\hline \multicolumn{5}{|l|}{ Sternopygidae } \\
\hline Eigenmannia virescens (Valenciennes, 1887) & $\mathrm{x}$ & $\mathrm{x}$ & & \\
\hline \multicolumn{5}{|l|}{ Pimelodidae } \\
\hline Pimelodus maculatus Lacépède, 1803 & $\mathrm{x}$ & $\mathrm{x}$ & & $\mathrm{x}$ \\
\hline Total de espécies identificadas & 5 & 7 & 4 & 3 \\
\hline Porcentagem da riqueza registrada na lagoa (\%) & 14,7 & 20,0 & 9,3 & 6,0 \\
\hline
\end{tabular}

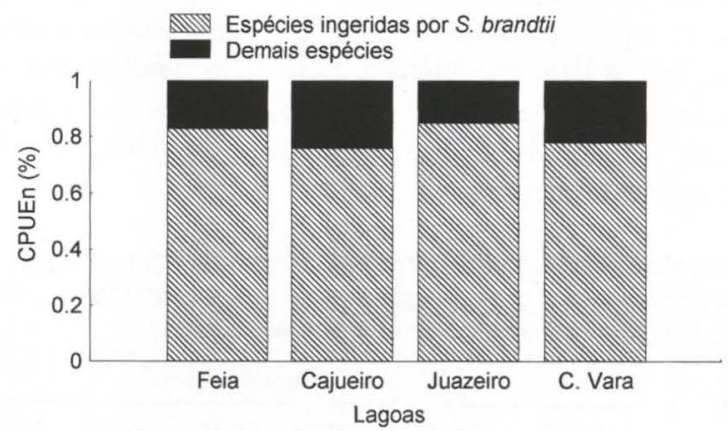

Fig. 1. Percentual das capturas por unidade de esforço em número (CPUEn) representado pelas espécies ingeridas por $S$. brandtii, em cada lagoa.

Em todas as lagoas foi observada marcada ontogenia trófica. Insetos aquáticos, principal item alimentar dos indivíduos menores, são substituídos, à medida em que crescem os indivíduos, por escamas e nadadeiras e, posteriormente, por pedaços de peixes e peixes inteiros (Fig. 3).

A regressão linear demonstrou que a freqüência de ocorrência de peixes no conteúdo estomacal foi diretamente proporcional à abundância de peixes das lagoas, calculada através da CPUEn (Fig. 4). O consumo de insetos aquáticos (IA $i$ para insetos aquáticos) foi maior na estação chuvosa, quando comparado coma a estação seca. (Fig. 5). 


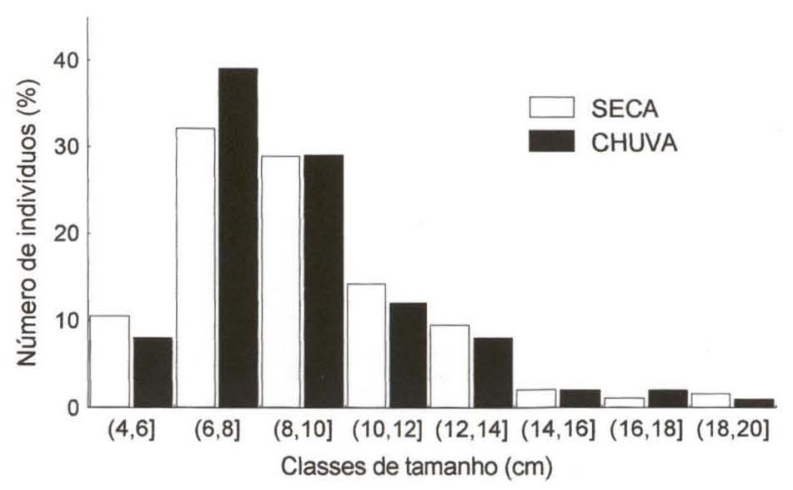

Fig. 2. Distribuição dos indivíduos de Serrasalmus brandtii com conteúdo estomacal por classe de tamanho nas estações seca e chuvosa.

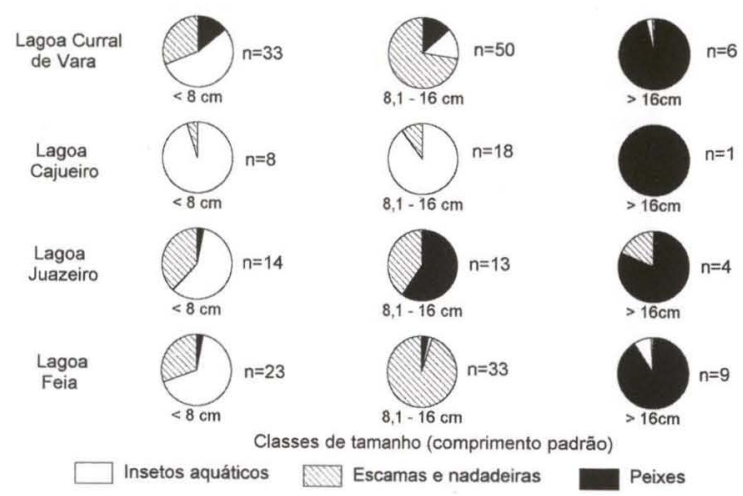

Fig. 3. Indice alimentar para os itens consumidos por Serrasalmus brandtii por lagoa e classe de tamanho.

\section{DISCUSSÃO}

A alimentação observada para $S$. brandtii, em todas as classes de tamanho, é predominantemente carnívora e coincide com a dieta observada por BEDÊ et al. (1993) na represa da Pampulha. Peixes fazem parte da dieta de inúmeras outras espécies que habitam as lagoas marginais do rio São Francisco, como o surubim Pseudoplatystoma coruscans (Agassiz, 1829); o dourado - Salminus brasiliensis (Cuvier, 1817); a traíra - Hoplias malabaricus (Bloch, 1794) e a piranha-vermelha - Pygocentrus piraya (Cuvier, 1820). No entanto, nas lagoas estudadas, predação mutilante, com a ingestão de pedaços de peixes, escamas e nadadeiras, parece ser praticada apenas por $S$. brandtii.

As espécies da subfamília Serrasalminae são únicas dentre os Characiformes por possuirem dentes e mandíbula bem adaptados para arrancar pedaços de carne de peixes maiores, ou mesmo de mamíferos (MYERS 1972; GERRY 1977). No entanto, estudos mais recentes têm revelado uma grande variedade de hábitos alimentares dentre as diversas espécies de piranha (GOULDING et al. 1988; SAZIMA \& MACHADO 1990). 


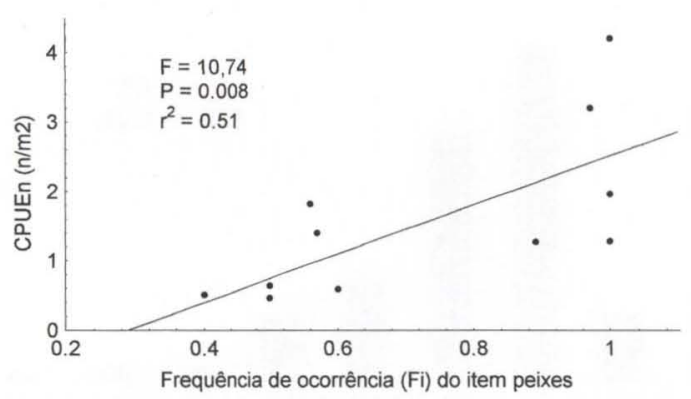

Fig. 4. Relação entre a freqüência de ocorrência ( $F i)$ de peixes, escamas e nadadeiras no conteúdo estomacal de Serrasalmus brandtii e a abundância de peixes nas lagoas (CPUEn).

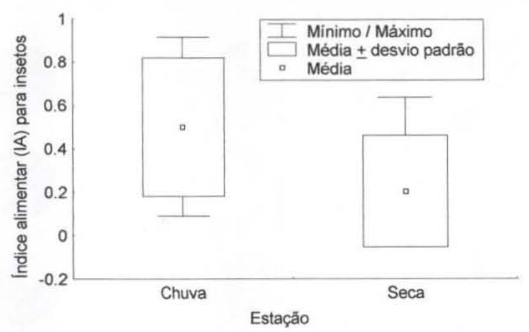

Fig. 5. Consumo de insetos aquáticos (IA para insetos aquáticos) por Serrasalmus brandtii nas estações seca e chuvosa.

Além de pedaços de peixes, S. brandtii ingeriu peixes inteiros, escamas, nadadeiras e insetos aquáticos. Até pouco tempo, poucos eram os relatos sobre a ocorrência de peixes inteiros no conteúdo estomacal de piranhas (LEÃo et al. 1991). Entretanto, trabalhos têm demonstrado que este pode ser um importante recurso alimentar para algumas espécies (NICO \& TAPHORN 1988; SAZIMA \& MACHADO 1990). A presença de nadadeiras e escamas no estômago tem sido relatada para inúmeras espécies (MACHADO-ALLISON \& GARCIA 1986; NICO \& TAPHORN 1988; BISTONI \& HARO 1995), constituindo o principal item alimentar de Serrasalmus elongatus, da bacia Amazônica (ROBERTS 1972). Frutos e sementes, considerados uma fração importante da alimentação de algumas espécies de piranhas (GOULDING 1980) não foram encontrados em nenhum estômago analisado.

Ontogenia trófica não parece ser um fenômeno raro entre espécies piscívoras, como as piranhas. NICO \& TAPHORN (1988) observaram que, em geral, o hábito alimentar e a dieta de oito espécies de piranhas dos llhanos da Venezuela, mudam com a idade, passando de mutilador de nadadeiras e/ou comedor de escamas quando jovem, até predominantemente piscívoro quando adulto. Além disto, indivíduos menores ingeriram, em grande parte, invertebrados. Ontogenia trófica também foi encontrada para nove espécies de piscívoros estudados por WINEMILLER (1989). Nestas, os jovens consomem insetos e microcrustáceos. A importância de invertebrados na alimentação de jovens de piranhas também foi descrita por SAZIMA \& ZAMPROGNO (1985). 
Diferenças sazonais na abundância de alimentos afetam diretamente as comunidades de peixes tropicais. Em resposta à estas variações, grande parte das espécies são oportunistas (LOWE-MCCONNELL 1975; 1987; WELCOMME 1985; NICO \& TAPHORN 1988), mudando de dieta de acordo com a disponibilidade de alimento (Lowe-MCCONNEL 1964; WeLCOMME 1985). Na estação chuvosa, por exemplo, algumas espécies passam a ingerir uma maior quantidade de invertebrados (PREJS \& PREJS 1987).

Os resultados indicam que as variações na dieta de $S$. brandtii estiveram relacionadas à abundância dos recursos alimentares. Além de se alimentar principalmente das espécies de peixes mais abundantes nas lagoas, o consumo deste item (incluindo escamas e nadadeiras) esteve relacionado com os valores das capturas em número nas lagoas, um indicativo do número de presas potenciais. Além disto, insetos aquáticos foram mais consumidos na estação chuvosa, período em que a temperatura da água, a superfície de colonização e o aporte de material alóctone aumentam (TAQUEDA et al. 1997), propiciando uma maior abundância de invertebrados aquáticos (WELCOMME 1985).

As diferenças sazonais na alimentação de $S$. brandtii poderiam ser derivadas de diferenças na estrutura etária da população, uma vez que a espécie apresenta ontogenia trófica. Entretanto, esta hipótese foi afastada, uma vez que as populações estudadas apresentaram a mesma estrutura em tamanho nas estações seca e chuvosa.

Assim, as mudanças sazonais e ontogenéticas observadas para $S$. brandtii sugerem que, apesar das especializações no aparato alimentar, estes peixes são oportunistas, como grande parte das espécies encontradas nas áreas alagáveis da América do Sul (Lowe-MCCONNELl 1975; WelCOMME 1985).

AGRADECIMENTOS. Aos amigos Fábio Vieira e Carlos Bernardo Mascarenhas Alves, Volney Vono, Gilmar Bastos Santos e Alexandre Lima Godinho pelas valiosas sugestões ao manuscrito. À CODEVASF, UFMG e CNPq, que possibilitaram a execução deste projeto.

\section{REFERÊNCIAS BIBLIOGRÁFICAS}

BedÊ, L.C; G.B. Santos; C.B.M. Alves \& A. Pelli. 1993. Alimentação de peixes do reservatório da Pampulha, Belo Horizonte, MG, com ênfase na malacofagia. Ceres 40: 429-437.

BIstoni, M.A \& J.G. HARO. 1995. Habitos alimentarios de Serrasalmus spilopleura (Pisces, Serrasamidae) en los bañados del río Dulce (Córdoba, Argentina). Rev. Brasil. Biol 55: 847-853.

BRaGA, A.R. 1964. Disponibilidade de peixes em poços do rio São Francisco. Bol.

Soc. Cear. Agron. 5: 77-86. - 1981. Ecologia e etologia das piranhas do nordeste do Brasil (Pisces

- Serrasalmus Lacépède, 1803). Fortaleza, Denocs, 268p.

GERY, J. 1977. Characoids of the word. Neptune, T.F.H. Publ., 672p.

GoUlding, M. 1980. The fishes and the forest. Explorations in Amazonian

natural history. Berkeley, University of California Press, 280p.

Goulding, M.; M.L. Carvalho \& E.G. Ferreira. 1988. Rio Negro: rich life in 
poor water. Hague, SBC Academic Publishing, 200p.

KAWAKAMI, E. \& G. VAZzoleR. 1980. Método gráfico e estimativa de índice alimentar aplicado ao estudo de alimentação de peixes. Bolm. Inst. Oceanogr., São Paulo, 29: 205-207.

LeÃo, E.L.M; R.G. Leite; P.T.C. Chaves \& E. Ferraz. 1991. Aspectos da reprodução, alimentação e parasitofauna de uma espécie rara de piranha, Serrasalmus altuvei, Ramíres, 1965 (Pisces, Serrasalmidae) do baixo rio Negro. Rev. Brasil. Biol 51: 545-553.

LOWE-MCCONNELL, R.L. 1964. The fishes of Rupunini savanna district of British Guiana, South America. Part 1: Ecological groupings of fish species and effects of the seazonal cycle on the fish. Jour. Limn. Soc., Zool., 45: 103-144. $337 \mathrm{p}$.

1975. Fish communities in tropical freshwaters. London, Longman,

1987. Ecological studies in tropical fish communities. London, Cambridge University Press, 382p.

Machado-Allison, A. \& C. GarCiA. 1986. Food habits and morphological changes during ontogeny in tree serrasalmin species of Venezuelan flood plain. Copeia 1: 93-96.

MyerS, J.S. 1972. The piranha book. Neptune, TFH Publ., 128p.

NICO, L.G. \& D.C. TAPHORN. 1988. Food habits of piranhas in the low llanos of Venezuela. Biotropica 20: 311-321.

PlanVASF. 1986. Plano Diretor para o Desenvolvimento do Vale do São Francisco. Brasília, Planvasf, 80p.

PAULY, D. 1994. Quantitative analysis of published data on the growth, metabolism, food consumption, and related features of the red-bellied piranha, Serrasalmus nattereri (Characidae). Environ. Biol. Fishes 41: 423-437.

PREJS, A. \& K. PREJS. 1987. Feeding of tropical freshwater fishes: seasonality in resource availability and resourse use. Oecologia 71: 397-404.

RoBERTS, T.R. 1972. Ecology of fishes in the Amazon and Congo Basins. Bull. Mus. Comp. Zool. 143: 117-147.

SAZIMA, I. \& C. ZAMPROGNO. 1985. Use of water hyacinths as shelter, foraging place, and transport by young piranhas, Serrasalmus spilopleura. Environ. Biol. Fishes 12: 237-240.

SAZIMA, I. \& F.A. MACHADO. 1990. Underwater observations of piranhas in western Brazil. Environ. Biol. Fishes 28: 17-31.

TAKEDA, A.M; G.Y. SHIMISU; J. HigUTI. 1997. Variações espaço-temporais da comunidade zoobentônica, p.157-177. In: A.E.M. VAZzolerM; A.A. Agostinho \& N.S. HAHn. A planície de inundação do alto rio Paraná: aspectos físicos, biológicos e socioeconômicos. Maringá, Eduem, 460p.

Welcomme, R.L. 1985. River Fisheries. FAO Fish. Tech Pap. 262: 1-330.

WINEMILLER, K.O. 1989. Ontogenetic diet shifts and resourse partioning among piscivorous fishes in the Venezuelan llanos. Environ. Biol. Fishes 26: 177-199.

Recebido em 06.VIII.1998; aceito em 05.XI.1999. 